\title{
Psychological and pedagogical competence of parents raising children included in joint education: theory and practice of research
}

\author{
Elena Slusareva*, Anna Kabushko, and Aleksej Dontsov \\ State budgetary educational institution of higher education «Stavropol state pedagogical \\ Institute», Russia
}

\begin{abstract}
The article presents theoretical approaches and an empirical study of the problem of psychological and pedagogical competence of parents of children included in joint education as conditions for the effective implementation of inclusive educational practice. Theoretical approaches to the definition of «psychological and pedagogical competence of parents», its structural components and their content, due to the specifics of joint education of children with different psychophysiological status, are considered. The results of an empirical study of the psychological and pedagogical competence of parents of two groups are presented: parents of children of primary school age with normal psychophysical development and parents of children with disabilities included in joint (inclusive) education. The reliability of the research results is due to the use of a complex of methods of theoretical and empirical research, the representativeness of the sample: 354 parents raising children included in joint education.
\end{abstract}

In the last decade, the term «joint (inclusive) education» has again firmly entered the psychological and pedagogical terminology, which has acquired a new meaning in connection with changes in educational practice. The very idea of inclusion is based on the concept of an «inclusive society».It means changing society and its institutions in such a way that they favor the inclusion of the "other» (a person of a different race, religion, culture, a person with disabilities) in society.Moreover, such a change in institutions is supposed that this inclusion contributes to the interests of all members of society (including individuals with disabilities), ensuring the equality of their rights.

If inclusion is not provided by a corresponding change in the educational process, then its result may be a deepening of the social maladaptation of children with disabilities and an increase in intolerance to them from those who do not have such restrictions. The readiness of the educational community to change towards the other is an important prerequisite for successful inclusion, and it must be brought up before a child with disabilities comes to school.

\footnotetext{
${ }^{*}$ Corresponding author: slusareva2005@yandex.ru
} 
The influence of the family on a growing child is stronger than all other educational influences. Only in the family many personality qualities are developed that cannot be brought up anywhere except the parental home.Therefore, parents must correctly, on a scientific and pedagogical basis, contribute to the success of their children in the system of joint (inclusive) education. And this is impossible without the psychological and pedagogical competence formed in parents in the field of inclusive education. And we are talking about all the parents whose children are included in the system of joint (inclusive) education.

Modern scholars interpret the psychological and pedagogical competence of parents as a broad general cultural concept that is part of the pedagogical culture (E.V. Bondarevskaya [2], Yu.A. Gladkova [3] and others); as a system of personality education: a set of personal and activity characteristics that determine the ability to effectively implement the process of raising a child in the family (S. S. Piyukova [7], V. V. Selina [8]); a set of personality and activity traits of the parent, which determine the ability to effectively implement the process of raising a child in the family and include: willingness and ability to perceive the child as a value; possession of basic psychological and pedagogical knowledge; the ability to search, perceive and select information.

The psychological and pedagogical competence of parents as a personal education integrates a combination of special knowledge and skills (knowledge of the methodological foundations and categories of pedagogy and psychology, understanding of the laws of socialization and personal development; an idea of the nature, goals and technologies of personal upbringing and education; understanding not only the laws of its anatomical and physiological, but also mental development at different age stages, etc.), methodological skills (the ability to independently find ways to solve complex parental problems, the skills of self-education and self-development as a parent).

The level of psychological and pedagogical competence of parents depends on the level of their education, general culture, individual characteristics and inclinations, determined by the level of their own upbringing, as well as accumulated life experience.Improving the psychological and pedagogical competence of parents raising children included in joint (inclusive) education should be considered in two aspects:increasing the parental competence of parents raising children with disabilities included in joint (inclusive) education;increasing the parental competence of parents raising children with normative psychophysical development, included in joint (inclusive) education.

Researchers note that successful education and upbringing of children with disabilities is impossible without a specific special education of parents. As noted by A.R. Muller, all the work of the institution's specialists should be aimed at ensuring that parents from passive observers become active participants in the upbringing and education of their children [5]. In turn, V.V. Tkacheva notes that the achievement of a psychocorrection effect is carried out as a result of the personal participation of the mother of the child with disabilities in his educational process [10]. G.A. Mishina is considering the forms of organization of the correctional and pedagogical work of a specialist-defectologist with a family raising a young child with impaired psychophysical development [6].She offers the following forms of organization: advisory and advisory; lecture and educational; organization of round tables, parent conferences; individual lessons with parents and their child; subgroup classes. E.A. Ekzhanova, offering group and individual forms of work with parents who have children with a mental retardation, recommends regular implementation of various types of interaction. Well established, according to E.A. Ekzhanova, such active forms of 
work with parents as workshops, counseling, psychological training, and psychological counseling [4].

At the same time, researchers note that the level of psychological and pedagogical competence of parents raising children with disabilities remains sufficiently low, which negatively affects the outcome of their education.

If the problems of the formation of psychological and pedagogical competence of parents raising children with disabilities are in the field of scientific and methodological study, then practically no attention is paid to the formation of the competence of parents raising children with normative development included in the joint education system. At the same time, there are studies that indicate the need to consider this problem (T.N. Adeeva, I.N.Simaeva, V.V. Khitryuk, etc.) $[1,9,11]$.

So, parents whose children were included in joint education often express fears that their child will not be able to accept their «unusual» peers, worry about conflicts between «typically» and «not typically developing» children, and don't know how to lead them themselves in cases of their occurrence. They fear that the presence in the classroom of children who require special support may delay the development of their own child, and they do not understand the advantages that their children have in interacting with children with disabilities - the development of personal qualities (tolerance, empathy, mutual assistance, etc.). The change in the attitude of parents raising children with normative development towards joint (inclusive) education should be promoted by the formation of psychological and pedagogical competence in them competency.In an inclusive educational environment, parents and parents of different categories of students combine and interact on the basis of respect for each other, understanding and acceptance of differences, their willingness to jointly teach children (I.N.Simaeva, V.V. Khitryuk) [9].

In its most general form, the concept of psychological and pedagogical competence of parents (or parental competence) in the field of inclusive education can be defined as the ability (availability of knowledge, skills, experience - «mastery of the way») and willingness (intention, emotional state, mood) to solve problems communication and interaction in various subject systems (V.V. Khitryuk) [11].

Summarizing the work of researchers in this field, we can distinguish the following structural components of this education:

1) the cognitive component (ideas about the nature of inclusive education, the features of interaction in an inclusive educational environment, special educational conditions for children with disabilities);

2) emotional component (attitude to inclusive educational processes, degree of satisfaction with the main characteristics of an inclusive educational environment);

3) conative (parental actions aimed at optimizing interaction in an inclusive educational environment).

These components and their indicators formed the basis of our empirical study.An empirical study was carried out in educational organizations that implement inclusive practices and are located in both urban and rural areas (Stavropol town, Novoaleksandrovsky district of Stavropol Region).

The study involved 273 parents raising children of primary school age with normal psychophysical development, of whom $35 \%$ had a higher education, those surveyed, $63 \%$ had an average education, and $2 \%$ had no education. In the future, in the text we will designate this group of parents as experimental group 1 (EG1).Of the 81 parents who raise children with disabilities (intellectual, sensory, motor disorders, disorders of the affective sphere), $17 \%$ of respondents have higher education, $47 \%$ have secondary education, and $36 \%$ do not have education. Most of them are represented by mothers - 
$94 \%$. In the future, in the text we will designate this group of parents as experimental group 2 (EG 2).

The total sample size is 354 parents of children included in co-education. The main research method is polling (questionnaires,conversation).The questionnaire proposes open, closed, and mixed-type questions to identify parents' perceptions of inclusive educational practices; attitude to coeducation, as well as their suggestions for optimizing the coeducation process.

The first criterion for the study was the presence of parents raising children included in joint education, ideas about inclusive educational processes, psychological characteristics of children with different psychophysiological status, educational conditions for teaching children (cognitive component of psychological and pedagogical competence of parents).

The questions of experimental methods were aimed at studying the opinion of two groups of parents about the optimal educational conditions for children with disabilities: comprehensive schools, special (correctional) educational organizations, individual (home) education.

Studying the opinion of parents regarding educational conditions for children with motor impairments, we received the following data: $35 \%$ of parents in the EG 2 believe that children with musculoskeletal impairments should study at a regular educational institution (mass school); whereas in EG 1 this opinion was shared by $28 \%$ of parents. «For» special (corrective) educational conditions 27\% of EG 2 and 37\% - EG 1 spoke in favor.

Hearing impaired children, according to the parents of EG 2, should study in general educational institutions ( $48 \%$ of parents) and special (correctional) institutions (45\%). We received more restrained answers regarding inclusive education for children with hearing impairments in EG 1: only $12 \%$ expressed positive relative co-education for this group of children and 52\% (most of the sample) considered the conditions for special (correctional) schools to be optimal.

We also note a similar point of view when choosing educational conditions for children with visual impairments: $45 \%$ of parents in the EG 2 consider the conditions of general educational organizations optimal and $41 \%$ consider them to be conditions of special (correctional) schools. Whereas in EG 1, 13\% of respondents had a positive attitude towards co-education of children with visual impairments. Most parents of the EG 1 consider special (correctional) conditions as the most optimal for visually impaired children $-49 \%$.

With regard to children with intellectual disabilities, the majority of parents of EG 2 $(86 \%)$ spoke in favor of special (corrective) learning conditions, while in EG 1 this opinion was shared by $56 \%$ of respondents. The idea of joint (inclusive) education of children with intellectual disabilities was supported by $9 \%$ of parents of EG 2 and 5\% of parents of EG 1. The remaining respondents recommended an individual form of education as optimal for this group of children.

A similar point of view for parents of both groups and for children with affective disorders: $52 \%$ of parents of the EG 2 and $77 \%$ of parents of group 1 consider special (corrective) educational organizations as the most optimal educational route for this category of children. $6 \%$ of parents of both groups consider co-educational opportunities; the rest is an individual educational route.

Thus, we note that if we are talking about children with severe impairments that limit the possibility of adaptation (social, psychological) in the educational environment (children with intellectual impairments and disorders of the affective sphere), then in this case the parents consider the conditions of special (corrective) schools and individual instruction. Whereas when it comes to children with sensory impairments 
(visually impaired, hearing impaired children) and motor impairments, parents find the conditions of general education and the conditions of special (correctional) schools equally attractive.

The next component of the study is the emotional component of psychological and pedagogical competence (the attitude of two groups of parents to joint education of children with different psychophysical status; satisfaction with the main characteristics of an inclusive educational environment).

Studying the attitude of the parents of experimental group 1 to the inclusion of children with disabilities in the educational process, we obtained the following results: the most representative are the responses about the positive attitude and acceptance $(60 \%) ; 23 \%$ expressed a sympathetic attitude and desire to help, and $3 \%$ made up answers that showed a negative attitude towards the process of joint learning of different groups of children. The leading feelings of parents of EG 1 towards children with disabilities are compassion (55\%), sympathy (68\%), and pity (61\%).

The percentage of parents who refrained from answering is rather high $(14 \%)$, which, in our opinion, may indicate the uncertainty of the position of parents in relation to inclusive educational processes. At the same time, it should be noted that the parents of EG 1 (36\% of the respondents) note that the attitude in children with disabilities can be related to the severity and severity of the child's disabilities that limit his ability to live, education and communicate.

No less interesting is the opinion of parents about how their children included in joint education relate to each other. Most parents of EG 2 (45\%) believe that peers with normal psychophysical development have a positive attitude towards children with disabilities included in co-education, the negative attitude was noted by the smallest number of respondents in EG $2-14 \%$, neutral $-42 \%$. We note similar indicators in EG1: the predominant answer was «benevolent attitude towards children with disabilities»- $46 \%$. At the same time, the response to rejection of children with disabilities is quite high $-22 \%$.

$15 \%$ of the parents of EG 1 noted that they observed cases of verbal use (ridicule, grazing, etc.) and less often - physical violence against children with disabilities. The facts of negative attitude towards their child with disabilities, on the part of peers noted $10 \%$ of parents of EG 2 (scoffed, laughed, teased); $57 \%$ of respondents deny this attitude to their child.

Our data confirm the data presented in the studies of T.N. Adeeva, E.S. Glukhova, S.A. Litvina,in which there is a contradictory tendency in the position of parents in relation to the included education: from positive, accepting attitude to hidden, rejected.

Studying the conative component of psychological and pedagogical competence (actions of parents aimed at optimizing the process of joint learning), we obtained the following results. Parents of the EG 1 as the primary actions indicated the need to create full psychological conditions in the educational institution (to perceive children with disabilities as all children, surround them with tolerance, understanding, etc.) $-12 \%$, and also indicated the need for material support, increase benefits for disability, improve public security $-30 \%$.

Parents of EG 2 redirected the emphasis of action to state and social structures. From these points of view, the optimization of joint (inclusive) training will be facilitated by such activities as: medical counseling $-67 \%$; psychological counseling $43 \%$; pedagogical counseling $-36 \%$; legal advice $-16 \%$; rental of household items $13 \%$; childcare and childcare $-7 \%$; purchase of goods on a preferential basis $-36 \%$.

The study allowed us to draw the following conclusions: the psychological and pedagogical competence of parents of children included in joint education is a systemic education, the structure of which includes: a cognitive component (knowledge and ideas 
of parents about an inclusive educational environment, special educational needs of children included in it, etc.); emotional (attitude to an inclusive educational environment and satisfaction with its characteristics); conative (behavior, actions of parents aimed at optimizing interaction in an inclusive educational environment).

The obtained empirical data allow us to note that in the structure of psychological and pedagogical competence of parents raising children with normal psychophysical development, the emotional component of psychological and pedagogical competence predominates: demonstrating the outwardly social acceptance of the idea of joint learning, they are not ready for an effective component - providing assistance and inclusion in interaction with subjects of an inclusive educational environment; consider special (correctional) conditions as the most appropriate for the special educational needs of children with disabilities. Whereas in the structure of psychological and pedagogical competence of parents of children with limited health abilities, the knowledge and experience of a parent interacting with their own child are reflected in the formation of the cognitive and emotional component of psychological and pedagogical competence with some deprivation of the conative component: insufficient involvement of parents in building an educational own child's route.

These features are due to both the position of parents in relation to coeducation and the position of specialists of a comprehensive school that implements inclusive educational practice, and require further scientific reflection and practical research.

\section{References}

1. T.N. Adeeva, Siberian Pedagogical Journal. 4, 112-118 (2016)

2. E.V. Bondarevskaya, Introduction to pedagogical culture (RGPI, Rostov-on-Don, 1995)

3. Yu.A. Gladkova, A differentiated approach to improving the psychological and pedagogical culture of the family in a preschool (2009)

4. E.A. Ekzhanova, Education and training of children with developmental disabilities.

1, 8-16 (2002)

5. A.R. Muller, Help for children with developmental disabilities: A book for parents (ARKTI, Moscow, 2000)

6. G.A. Mishina, Ways of forming cooperation of parents with young children with developmental disabilities (Moscow,1998)

7. S.S. Piyukova, Formation of pedagogical competence of foster parents (Moscow, 2003)

8. V.V. Selina, Bulletin of NovSU. 45, 68-70 (2008)

9. I.N. Simaeva, Bulletin of the Baltic Federal University named after I. Kant. 11, 54-62 (2015)

10. V.V. Tkacheva, Defectology. 3, 30-36 (1999)

11. V.V. Khitryuk, Special Education: Materials of the XII Intern. scientific Conf., April 21-22. 249-253 (2016) 